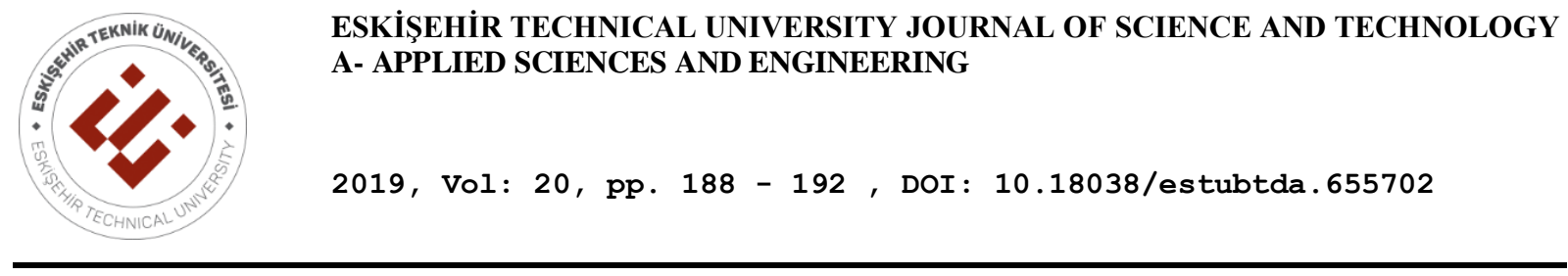

\title{
DYNAMICAL FRACTAL ANALYSIS OF THE ACOUSTIC ULTRA-WIDEBAND SIGNAL CAUSED BY THE CHELYABINSK METEOROID
}

\author{
Andriy ONISHCHENKO ${ }^{1, *}$, Leonid CHERNOGOR ${ }^{2}$, Oleg LAZORENKO ${ }^{3}$ \\ ${ }^{1}$ Physics Department, Faculty of Automatics and Computerized Technologies, \\ Kharkiv National University of Radioelectronics, Kharkiv, Ukraine \\ ${ }^{2}$ Space Radio Physics Department, School of Radiophysics, Biomedical Electronics and Computer Systems, \\ V. N. Karazin Kharkiv National University, Kharkiv, Ukraine \\ ${ }^{3}$ General Physics Department, School of Physics, V. N. Karazin Kharkiv National University, Kharkiv, Ukraine
}

\begin{abstract}
The non-linear paradigm clams, that many processes in open, non-linear, dynamical systems, which caused by the nonstationary, powerful sources, are appeared to be short-time, non-linear, ultra-wideband and fractal ones. The acoustic signals created by the Chelyabinsk meteoroid fall were shown to be namely ones of them. Using a new fractal analysis method called as 'Dynamical Fractal Analysis', the fractal properties of these signals were investigated. The corresponding numerical characteristics were estimated and discussed.
\end{abstract}

Keywords: Chelyabinsk meteoroid, Acoustic signal, Ultra-wideband process, Non-linear paradigm, Fractal analysis, Nonstationary fractal structure

\section{INTRODUCTION}

Being formulated in the late 1970s by prof. L. F. Chernogor, the non-linear paradigm claims, that many processes in open, non-linear, dynamical systems, which caused by impact of a non-stationary, powerful source, are appeared to be short-time, non-linear, ultra-wideband (UWB) and fractal ones [1 - 3]. The Chelyabinsk meteoroid fall is namely such source, and the Earth - atmosphere - ionosphere magnetosphere (EAIM) system is namely such system. Therefore, it is not surprising, that using so called 'System Spectral Analysis', the acoustic signals generated in the EAIM system by the Chelyabinsk meteoroid fall were shown to be UWB ones $[4,5]$. Moreover, the corresponding perturbations in the EAIM system were appeared to be global. This fact is fully explained by the phenomenon of appearance of the large-scale and global perturbations in the EAIM system caused by a non-stationary, powerful source influence. This phenomenon had been discovered in the former Soviet Union in the late 1980s by the scientific group led by prof. L. F. Chernogor [1 - 3].

At the same time, according the non-linear paradigm, the acoustic UWB signals listed above may be the fractal UWB (FUWB) signals. Therefore, the theme of the paper seems to be actual, topical and interesting.

The purpose of this paper is to investigate the fractal properties of these signals with usage of a new fractal analysis method, which is able to research a non-stationary fractal structure of a signal.

\section{CHELYABINSK METEOROID FALL EVENT}

The space body fall took place in Chelyabinsk region (Russia) February 15, 2013 and is known as 'Chelyabinsk meteoroid'. One of a large number of very complex processes in the EAIM system

*Corresponding Author: andrey.onishchenko@nure.ua

Received: 14.10.2019 Published:16.12.2019 
generated during this event is the infrasonic waves. Being very powerful, these acoustic signals were detected by the stations of both the international system of monitoring of nuclear tests and the geophysical observatories. It is necessary to point, that amplitude and spectrum of the infrasound signals carry a lot of information about the source of the waves and the atmosphere parameters (see, for example, $[6-9])$.

The infrasonic signals generated by the Chelyabinsk meteoroid fall and investigated in this paper were detected by the sensitive microbarographs of the IS27 station (Germany) placed in the Antarctica.

Overcame the distance about $15500 \mathrm{~km}$, first of them (the direct signal) was registered February 15, 2013 between 17.30 UT and 18.15 UT. The second signal (the reverse signal) passed a greater distance (about $25000 \mathrm{~km}$ ) and therefore was received some later (February 16, 2013 between 02.50 UT and 03.35 UT). At the Figure 1, a and the Figure 2, a both the signals are shown.

\section{DYNAMICAL FRACTAL ANALYSIS METHOD BASES}

If a signal has complex, non-stationary fractal structure, the Hurst exponent calculated for whole signal is not able to describe this. at all [10]. The usage of a time domain window is needed. But a Hurst exponent value estimated in such way will depend from the window width. We propose a new idea to choice a window width, which may be considered as an optimal (in some sense) for a signal or for its part. First, it is necessary to estimate the fractal dimension $D_{H}$ based on the Hurst exponent (in bounds of Generalized Brownian Motion Model they connected with $D_{H}=2-H$ ) with sliding time domain window with changing width $T$. In such way we obtain $D_{H}$ distribution at the time-period plane $(t, T)$. Second, we use module, angle and angle skeleton of the complex valued spectral function (SF) of the analytic wavelet transform (AWT) [11]. Researching the time-period structures of the signals, some fixed $T$ values are obtained. Third, for these $T$ values the dependences $D_{H}(T)$ are boiled and $D_{H}$ values for each signal or each signal part are estimated. Thus, such approach we call as 'Dynamical Fractal Analysis' (DynFA).

\section{ANALYSIS RESULTS AND DISCUSSION}

According to the SSA result $[4,5]$, there are two UWB processes with changing mean frequency (ChMF) in both the direct (Figure 1, a) and the reverse (Figure 2, a) signals. In the direct signal the first ChMF UWB process started approximately at $17.41 \mathrm{UT}(t=675 \mathrm{~s})$, its duration was $\tau=600-660 \mathrm{~s}$, its wideband index was $\mu=0,8$, the dynamical wideband index (for window width $\Delta t_{w}=270 \mathrm{~s}$ ) was changed in bounds $\mu_{d}=0,6-0,3$ with time, the disturbance periods of the signal were $T=35-90 \mathrm{~s}$, the mean period $T_{0}$ was decreased approximately linearly from $65 \mathrm{~s}$ to $40 \mathrm{~s}$. This information obtained in the SSA with the non-linear transforms usage can be seen at the Figure 1, h, but with bad quality due to much worse time-frequency resolution of the AWT. The DynFA shows, that the fractal dimension $D_{H}$ of this process vary in bounds $1,38-1,59$ (Figure $1, \mathrm{~d}$ ).

For the second ChMF UWB process started first of them started approximately at 17.52 UT $(t=1300$ s), we had $\tau=900-960 \mathrm{~s}, \mu=0,8, \mu_{d}=0,2-0,3, T=20-60 \mathrm{~s}$, the mean period $T_{0}$ was decreased approximately linearly from $50 \mathrm{~s}$ to $30 \mathrm{~s}$. With DynFA we obtain, that $D_{H}=1,51-1,78$ (Figure 1, f).

For the first ChMF UWB process started approximately at $03.03 \mathrm{UT}(t=785 \mathrm{~s})$ in the reverse signal, we obtained $\tau=600-900 \mathrm{~s}, \mu=0,7, \mu_{d}=0,35-0,3, T=20-40 \mathrm{~s}$, the mean period $T_{0}$ was decreased approximately linearly from $32 \mathrm{~s}$ to $22 \mathrm{~s}$. The DynFA results show that $D_{H}=1,45-1,77$ (Figure 2, f). 
Second ChMF UWB process started approximately at $03.10 \mathrm{UT}(t=1190 \mathrm{~s})$ had $\tau=1500 \mathrm{~s}, \mu=0,8, \mu_{d}$ $=0,5-0,2, T=28-70 \mathrm{~s}$, the mean period $T_{0}$ was decreased approximately linearly from $55 \mathrm{~s}$ to $30 \mathrm{~s}$. The DynFA gives $D_{H}=1,61-1,77$ (Figure 2, f).

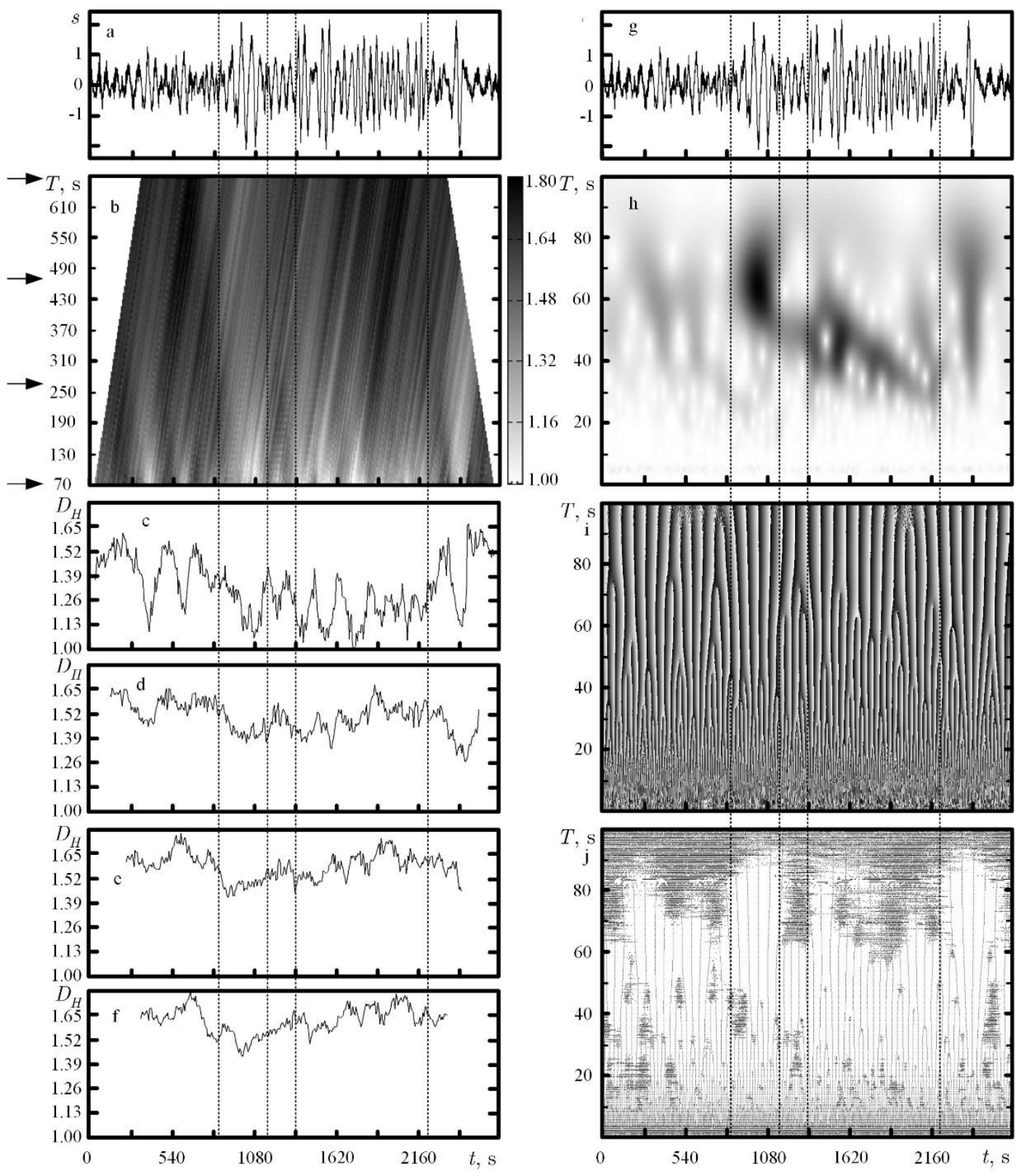

Figure 1. The DynFA results of the acoustic UWB signals generated by the Chelyabinsk meteoroid fall for direct signal (February 15, 2013): (a), (g) The signal in time domain; (b) The signal fractal dimension $D_{H}$ based on the Hurst exponent at the time - period plane $(t, T)$; (c) The signal fractal dimension $D_{H}$ with time $t$ for the time-domain window with fixed width $T=70 \mathrm{~s}$; (d) The same for $T=270 \mathrm{~s}$; (e) The same for $T=$ $470 \mathrm{~s}$; (f) The same for $T=670 \mathrm{~s}$; (h) The AWT SF module obtained with complex Morlet wavelet cmor 11.5; (i) The AWT SF angle obtained with complex Morlet wavelet cmor1-1.5, (j) The AWT SF angle skeleton obtained with complex Morlet wavelet cmor1-1.5. The dotted lines help to divide a signal investigated at some parts, for each of the fractal dimensions $D_{H}$ values are estimated. The arrows show the fixed $T$ values, for which $D_{H}(T)$ dependences are built. 
Most likely, the first UWB process in each signal pair may be explained by generation and propagation of the explosive shock wave. Second UWB process may be connected with generation and propagation of the ballistic wave.

It is important to point, that the signals analyzed contain a lot of information about as their source, as the state of the environment along the signal propagation path.

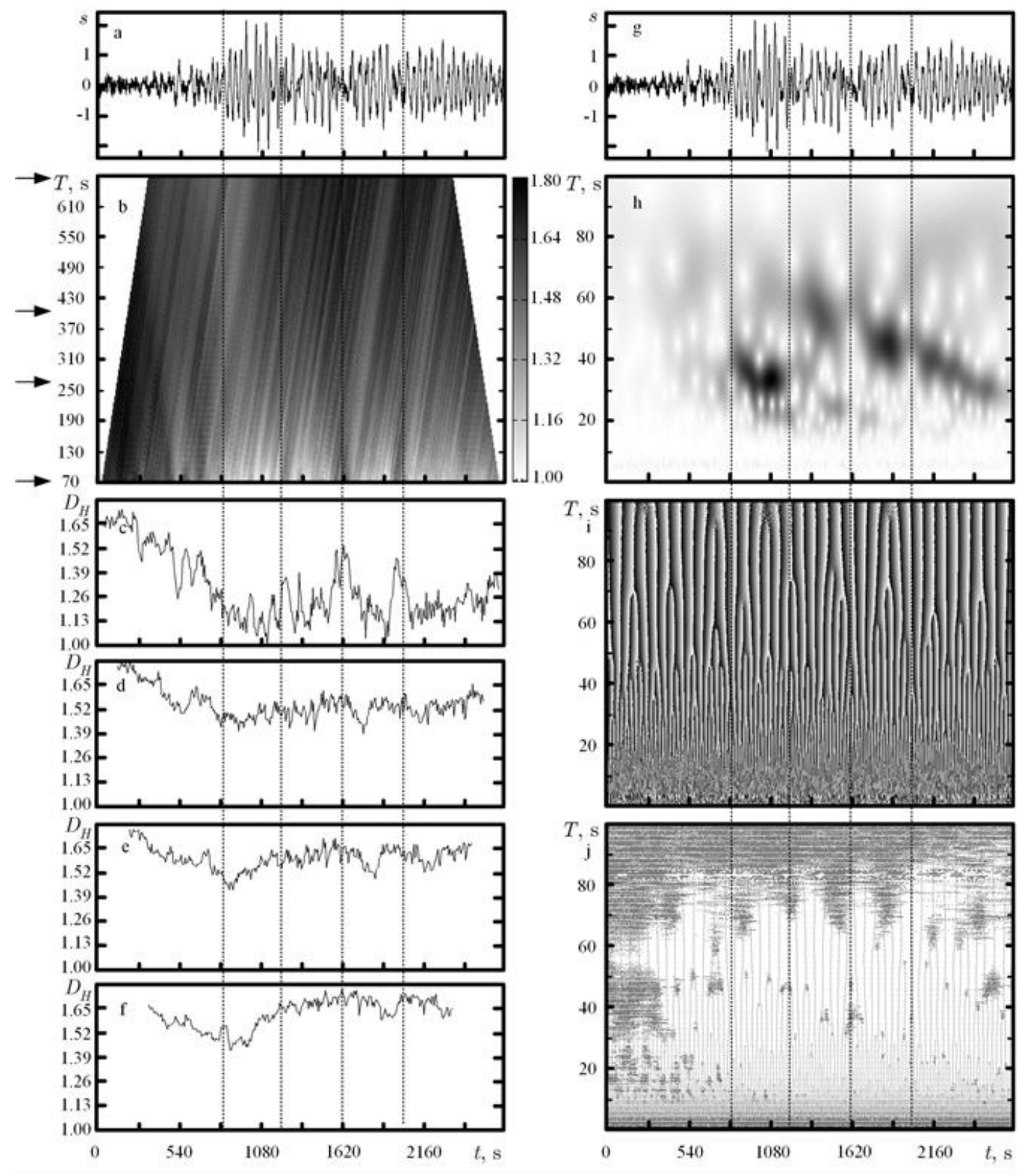

Figure 2. The DynFA results of the acoustic UWB signals generated by the Chelyabinsk meteoroid fall for reverse signal (February 16, 2013): (a), (g) The signal in time domain; (b) The signal fractal dimension $D_{H}$ based on the Hurst exponent at the time - period plane $(t, T)$; (c) The signal fractal dimension $D_{H}$ with time $t$ for the time-domain window with fixed width $T=70 \mathrm{~s}$; (d) The same for $T=270 \mathrm{~s}$; (e) The same for $T=$ $470 \mathrm{~s}$; (f) The same for $T=670 \mathrm{~s}$; (h) The AWT SF module obtained with complex Morlet wavelet cmor 11.5; (i) The AWT SF angle obtained with complex Morlet wavelet cmor1-1.5, (j) The AWT SF angle skeleton obtained with complex Morlet wavelet cmor1-1.5. The dotted lines help to divide a signal 
investigated at some parts, for each of the fractal dimensions $D_{H}$ values are estimated. The arrows show the fixed $T$ values, for which $D_{H}(T)$ dependences are built.

\section{CONCLUSIONS}

The acoustic UWB signals generated by the Chelyabinsk meteoroid fall were found to be the fractal ChMF UWB processes with complex, non-stationary fractal structure.

Using a new fractal analysis method created specially and called as the 'Dynamical Fractal Analysis', the non-stationary fractal properties of the acoustic signals generated by the Chelyabinsk meteoroid fall were investigated and corresponding numerical characteristics were estimated.

\section{REFERENCES}

[1] Chernogor LF. About Nonlinearity in Nature and Science: Monograph. Kharkov: V. N. Karazin Kharkov National University, 2008 (in Russian).

[2] Chernogor LF, Rozumenko VT. Earth - Atmosphere - Geospace as an Open Nonlinear Dynamical System. Radio Phys and Radio Astron 2008, 13, 2: 120-137.

[3] Chernogor LF. The Earth - Atmosphere - Geospace Environment System as an Open Dynamic Nonlinear One. Space Sci and Tech 2003, 9, 5/6: 96-105 (in Russian).

[4] Chernogor LF, Lazorenko OV. System Spectral Analysis of the Ultra-Wideband Signals Caused by the Chelyabinsk Meteoroid. In: 8th International Conference on Ultrawideband and Ultrashort Impulse Signals; 5-11 September 2016; Odessa, Ukraine. Kharkiv, Ukraine: IEEE, 85-88.

[5] Lazorenko OV, Chernogor LF. System Spectral Analysis of Infrasonic Signal Generated by Chelyabinsk Meteoroid. Radioelect and Comm Sys 2017, 60, 8: 331-338.

[6] Edwards WN. Estimates of meteoroid kinetic energies from observations of infrasonic airwaves. Atmos. Solar-Terr. Phys. 2006, 68: 1136-1160.

[7] Ens TA, Brown PG, Edwards WN, Silber EA. Infrasound production by bolides: A global statistical study. Atmos. Solar-Terr. Phys. 2012, 80: 208-229.

[8] Le Pichon A, Ceranna L, Pilger C. Russian fireball largest ever detected by CTBTO infrasound sensors. Geophysical Research Letters 2016, 40, 14: 3732-3737.

[9] Chernogor LF, Rozumenko VT. The physical effects associated with Chelyabinsk meteorite's passage. Problems of Atomic Science and Technology 2013, 86, 4: 136-139.

[10] Feder J. Fractals. New York: Plenum Press, 1988.

[11] Mallat S. A wavelet tour of signal processing. San Diego, London, Boston, N.Y., Sydney, Tokyo, Toronto: Academic Press, 1998. 